\title{
Winter sampling and seasonal variation in litter- dwelling beetle assemblages using a sifting method
}

\author{
Ui-Joung Byeon ${ }^{1}$, Seung-Il Lee ${ }^{2 \dagger}$, Sun-Jae Park ${ }^{3}$, A-Young Kim ${ }^{3}$, and Jong-Seok Park ${ }^{1 \star}$ (D) \\ ${ }^{1}$ Department of Biological Sciences and Biotechnology, Chungbuk National University, 1 Chungdae-ro, Seowon-gu, \\ Cheongju-si, Chungbuk-do 28644, Republic of Korea, ${ }^{2}$ Natural Resources Canada, Canadian Forest Service, Northern \\ Forestry Centre, 5320-122 Street, Edmonton, Alberta, T6H 3S5, Canada, and ${ }^{3}$ Animal Resources Division, National \\ Institute of Biological Resources, Incheon 22689, Republic of Korea \\ ${ }^{\star}$ Corresponding author. Email: jpark16@cbnu.ac.kr
}

(Received 17 March 2021; accepted 12 August 2021)

\begin{abstract}
Litter-dwelling arthropods play an important role in maintaining forest ecosystem function. This study was designed to understand seasonal variations and diversity of litter-dwelling adult beetles, one of the most diverse groups of arthropods. Sampling was conducted in mixed-wood forests of South Korea between March and December 2019, covering all seasons, including winter. We used a sifting method and a Berlese funnel to collect arthropods living in leaf litter and soil. We collected a total of 5820 invertebrates representing six orders, of which 1422 were beetles representing 24 families and minimum 141 species. Beetle species richness was highest in spring and lowest in summer based on rarefaction and extrapolation. However, beetle abundance was lowest in spring, but abundance was similar among the other seasons. Beetle assemblage composition was correlated significantly with soil surface and atmospheric temperature. The assemblage composition differed among seasons, except between spring and winter, which overlapped slightly. The combined sifting-Berlese funnel method showed great advantages for investigating the diversity of overwintering arthropods. Continued study of the relationship between arthropods and the leaf-litter environment is essential to understand this microecosystem and will increase the chance of discovering new beetle species.
\end{abstract}

\section{Introduction}

Arthropods account for more than $80 \%$ of animal species, among which litter-dwelling invertebrates account for a large proportion of the world's biodiversity (Ødegaard 2000). They represent important food sources for many vertebrates and other predatory invertebrates (Pianka and Parker 1975; Redford 1987; McNabb et al. 2001). Beetles (Coleoptera) are among the most diverse group of arthropods (Hammond 1992; Rosenzweig 1995), live in a variety of habitats, and have a wide diet spectrum, from plant leaves, tree sap, animals, fungi to dead or decaying wood (Cott 1940; Ruppert et al. 2004). They play an important role in forest ecosystem services, recycling organic components and nutrients, affecting energy flow, and so on (Giller 1996; Artz et al. 2010; Roy et al. 2018).

${ }^{\dagger}$ Current address: Department of Renewable Resources, University of Alberta, 771 General Services Building, Edmonton, Alberta, T6G 2H1, Canada; Alberta Biodiversity Monitoring Institute, Edmonton, Alberta, T6G 2H1, Canada.

Subject editor: Jeff Battigelli

(C) The Author(s), 2021. Published by Cambridge University Press on behalf of the Entomological Society of Canada. This is an Open Access article, distributed under the terms of the Creative Commons Attribution licence (https://creativecommons.org/licenses/by/4.0/), which permits unrestricted re-use, distribution, and reproduction in any medium, provided the original work is properly cited. 
Leaf litter is an important environment, harbouring many organisms, where interactions between litter-dwelling insects and the environment are ecologically important (Moore et al. 1988; Hughes et al. 2000; Jeffery and Gardi 2010). As leaf litter decomposes, it releases nutrients and moisture, promoting fungal growth and sporulation (Kerekes et al. 2013; Krishna and Mohan 2017). Litter-dwelling arthropods use this nutrient-rich environment and interact with each other (Seastedt and Crossley 1984). Leaf litter creates a variety of soil microhabitats that contain food resources, and it protects organisms from poor weather conditions and predators (Hamilton 2015). These microhabitats vary throughout the year and affect the availability of nutritional resources for species belonging to different feeding guilds (Fittkau and Klinge 1973; Hättenschwiler et al. 2005).

Changes in season or temperature affect the diversity and abundance of adult beetles that reside in leaf litter and soil (Pinheiro et al. 2002). The response of beetles to these changes differs worldwide, especially between temperate and tropical zones (Wolda 1978a, 1978b, 1980; Wolda and Broadhead 1985; McElravy and Resh 1987). In the temperate zone, where there are four seasons, for example, different species are active in different seasons (Scott and Epstein 1987). Summer in temperate regions is hot and humid, and the days are long. These environmental conditions are expected to increase the activity of adult beetles and increase their diversity and abundance (Whittaker and Tribe 1998; Frazier et al. 2006; Deutsch et al. 2008). Conversely, temperate-zone winters are cold and dry, with a short photoperiod, leading to decreased activity by adult beetles and a decline in species diversity and abundance (Mellanby 1939; Taylor 1963; Wolda 1988). Unfavourable cold environments also cause many insects to delay their development (Tauber et al. 1986; Saunders 2002), and many beetles enter diapause in an adult stage for winter (Danks 1987). Most adult beetles are sensitive to these environmental changes, and thus monitoring changes in diversity, abundance, and community structures of these organisms is highly important for biodiversity conservation (Agosti et al. 2000; Hilty and Merenlender 2000; Kime and Golovatch 2000; Longcore 2003; Bouyer et al. 2007; Siddig et al. 2016).

Several studies in Korea have investigated seasonal changes in insect biodiversity, including adult beetles. However, these studies have limited sampling periods and usually exclude the winter season. Furthermore, pitfall traps, light traps, or sweeping methods were typically used to collect insects (Lee et al. 2005; Byun et al. 2009a, 2009b, 2010; Lee et al. 2009; Park et al. 2010; Park et al. 2011; Lim et al. 2012; Kim and Kwon 2016). In the present study, we combined a sifting technique with Berlese funnel extraction to collect samples of litterdwelling arthropods during all seasons because of the limited capability of other trapping methods for sampling overwintering arthropods. Because Berlese funnels direct constant high temperature and light towards litter samples, they can provide a strong signal to awaken insects from diapause (Saunders 2002). In this way, the use of Berlese funnels has the potential to collect insects and other soil arthropods in the winter season.

Few studies have addressed the full seasonal spectrum of litter-dwelling animals and their relationships with the environment. The present study investigated seasonal variations and diversity of litter-dwelling adult beetle assemblages. It is the first study to sample beetles using a sifting method in all seasons, including winter, in the temperate forests of Korea. For this study, we predicted that (1) adult beetle species richness and abundance would be highest in summer and lowest in winter because many adults may not overwinter in leaf litter (Leather et al. 1995) and (2) the adult beetle assemblage composition would differ among seasons because different species have developed various life-history strategies to deal with seasonality (Tauber and Tauber 1976; Wolda 1988). The results will provide important baseline data related to seasonal variations in the biodiversity of litter-dwelling beetles. 


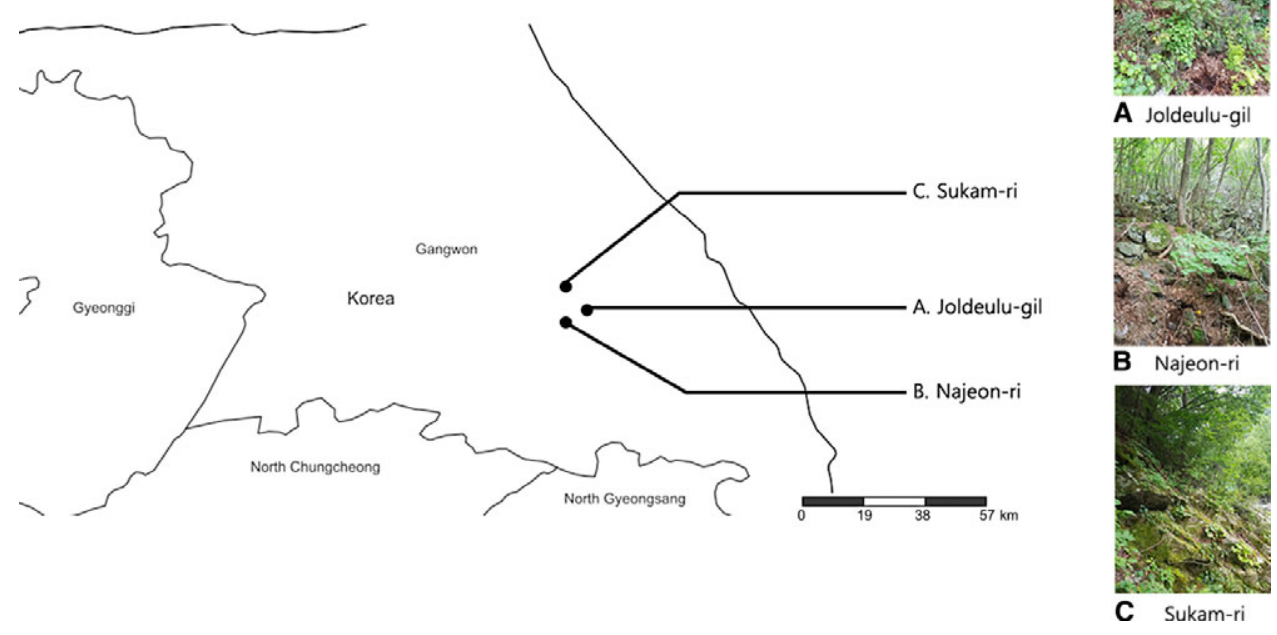

Fig. 1. Study sites in Bukpyeong-myeon, Jeongseon-gun, Gangwon-do, South Korea: A, Joldeulu-gil (37 $26^{\prime} 53.8^{\prime \prime} \mathrm{N}$, $128^{\circ} 38^{\prime} 20.9^{\prime \prime}$ E, 384 m elevation); B, Najeon-ri ( $37^{\circ} 26^{\prime} 02.0^{\prime \prime} \mathrm{N}, 128^{\circ} 36^{\prime} 57.0^{\prime \prime} \mathrm{E}, 782 \mathrm{~m}$ elevation); and C, Sukam-ri (37 29' 43.0" N, $128^{\circ} 35^{\prime} 00.0^{\prime \prime}$ E, 450 m elevation).

\section{Study sites}

\section{Materials and methods}

This study was conducted in Bukpyeong-myeon, Jeongseon-gun, Gangwon-do, South Korea, which is located in the southeastern part of Gangwon-do and the centre of the Mt. Taebaek Range. The average annual temperature in Jeongseon-gun is about $11^{\circ} \mathrm{C}$. August is the hottest month, with an average temperature of about $30^{\circ} \mathrm{C}$, and January is the coldest month, with an average temperature of $-9.6^{\circ} \mathrm{C}$. Annual rainfall averages about $1100 \mathrm{~mm}$, most of which falls in the summer (Korea Meteorological Administration 2020).

We selected three sites (site 1: Joldeulu-gil (Jol), 384 m elevation, $37^{\circ} 26^{\prime} 53.8^{\prime \prime} \mathrm{N}, 128^{\circ} 38^{\prime} 20.9^{\prime \prime} \mathrm{E}$; site 2: Najeon-ri (Na), 782 m elevation, $37^{\circ} 26^{\prime} 02.0^{\prime \prime} \mathrm{N}, 128^{\circ} 36^{\prime} 57.0^{\prime \prime} \mathrm{E}$; and site 3: Sukam-ri (Suk), $450 \mathrm{~m}$ elevation, $37^{\circ} 29^{\prime} 43.0^{\prime \prime} \mathrm{N}, 128^{\circ} 35^{\prime} 00.0^{\prime \prime} \mathrm{E}$; Fig. 1). All sample areas were mixed-wood forests containing five dominant tree species: Quercus mongolica Fischer ex. Ledebour (Fagaceae), Q. serrata Murray (Fagaceae), Pinus densiflora Siebold \& Zuccarini (Pinaceae), P. koraiensis Siebold \& Zuccarini (Pinaceae), and Betula platyphylla Sukaczev (Betulaceae) (Heo and Lee 2015). At each site, we sampled soil and deciduous leaf litter near stream margins. The largest distance between sites was $6 \mathrm{~km}$ (between Jol and Suk), and the two closest sites were $2 \mathrm{~km}$ apart (Jol and $\mathrm{Na}$ ).

\section{Sampling methods}

We sampled leaf litter once each season, March (spring), August (summer), October (autumn), and December (winter) 2019. Each seasonal collection was carried out for $1 \sim 2$ days. A total of 31 samples were collected: seven samples each in spring, summer, and fall, and 10 samples in winter. To sample the frozen ground in winter, we first sifted the upper leaf litter and then tried to dig up the upper part of frozen soil that enabled the soil sample to be sifted. Although we collected more litter samples in winter than in the other seasons, the total volume of the samples was similar for each season and each location. 
Fig. 2. Sifter and fabric bag: $\mathbf{A}$, top view of the $25 \times 25$-cm-square, $1 \times 1$-cm-mesh sieve and $\mathbf{B}$, side view.

A

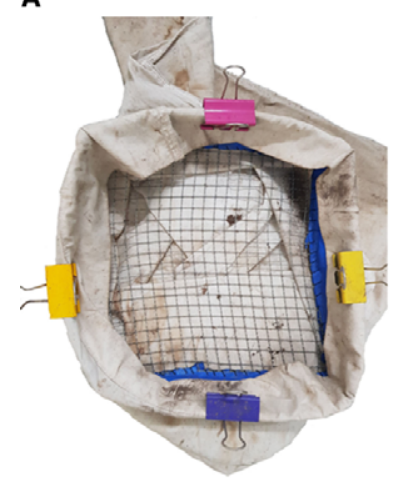

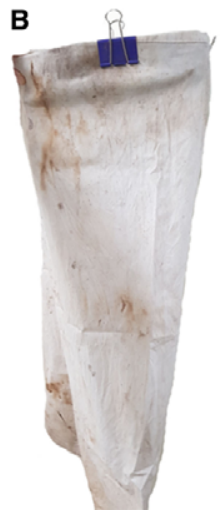

A

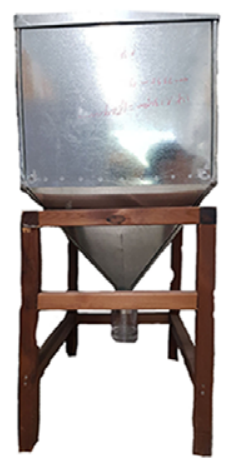

B

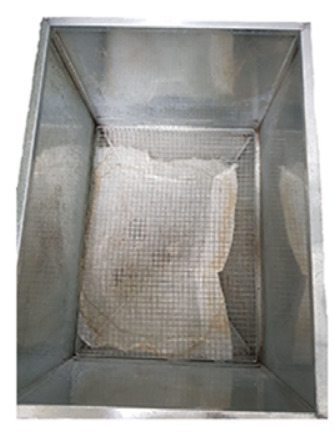

C

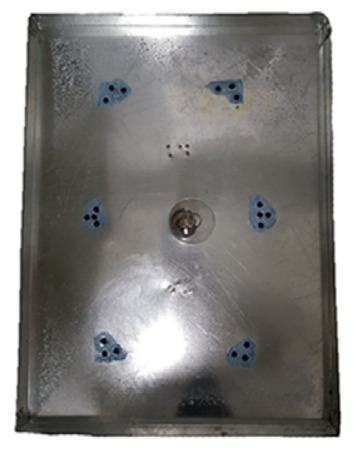

Fig. 3. Berlese funnel: A, external shape of the Berlese funnel attached to the wooden support frame; B, inside of the 42-cm-wide, 57-cm-long and 36-cm-high funnel; and C, the funnel's inner lid connected to a 100-Watt light bulb.

To standardise sifting, 3-4 L of mixed litter samples from Pinus and Quercus species were collected during each sampling using a sifter, which consisted of a $25 \times 25$-cm-square, 7-cm-deep, $1 \times 1-\mathrm{cm}$-mesh sieve that fed into a fabric bag (Fig. 2). Small fragments of leaves and soil that included arthropods dropped into the fabric bag when leaf litter was shaken in the sifter. The sieved samples were brought to the laboratory in the fabric bags and placed in Berlese funnels to collect arthropods.

Berlese funnels take advantage of soil arthropods' behaviour to avoid light and dry environments, which, in the case of the funnel method, causes both overwintering and nonoverwintering arthropods to move earthwards out of litter samples and into a collection chamber below. The Berlese funnels used in the present study were constructed of tin, which does not rust, and measured $42 \mathrm{~cm}$ wide, $57 \mathrm{~cm}$ long, and $36 \mathrm{~cm}$ high. A piece of $30 \times 40-\mathrm{cm}$ rectangular $0.1 \times 0.1-\mathrm{mm}$-mesh gauze was attached to a $1 \times 1-\mathrm{cm}$ metal sieve and placed inside the funnel. The funnel's top lid contained a 100-Watt incandescent bulb to emit light and heat. We operated the Berlese funnel for about a week for each litter sample. Arthropods dropped to the bottom of the funnel and were placed in a collecting jar with $70 \%$ ethanol (Fig. 3).

Whenever possible, adult beetles were identified to species level. However, due to the lack of taxonomic information on litter-dwelling beetles in Korea, some species were identified only to the genus level or multiple species were combined as a single species group and treated as one species for analysis (e.g., Aleocharinae spp.). Adult beetles were identified using the keys in 
taxonomic literature (e.g., Hayashi et al. 1984; Kurosawa et al. 1985; Ueno et al. 1985; Kurbatov 1991; Cho and Ahn 1999, 2001; Arnett and Thomas 2000; Kim and Ahn 2000a, 2000b; Park et al. 2012; Lee and Ahn 2015, 2019; Ahn et al. 2017; Kim et al. 2017; Jeong and Ahn 2018; Choi et al. 2020; Hoshina and Park 2020). We used a Garmin GPSmap 60CSx (Garmin Ltd., Schaffhausen, Switzerland) to measure geographic position and altitude, a Tenmars TM-183 meter to measure temperature and humidity (Tenmars Electronics Co., Taipei, Taiwan), and a Bosch Professional GIS 500 thermo detector (Bosch, Gerlingen, Germany) to measure soil temperature at each sampling site. Most specimens used for the study were deposited in the Chungbuk National University Insect Collection (Cheongju, South Korea).

\section{Statistical analyses}

We used permutational multivariate analysis of variance to test differences in species composition among season $\times$ location combinations (Anderson et al. 2008). Bray-Curtis distances were calculated on square root transformed data, with 9999 permutations for the main test. We partitioned variation using the default type III sum of squares (Anderson et al. 2008). When the main tests were significant $(P<0.05)$, we performed 999 permutations for an a posteriori pairwise comparison. We used the PERMANOVA+ add-on package for Primer, version 7, for these analyses (Anderson et al. 2008; Clarke and Gorley 2015).

We used redundancy analysis (1) to visually understand the structures of adult beetle assemblage among season $\times$ location combinations and (2) to determine the significant environmental variables underlying the variations in adult beetle assemblages among the above combinations. This method represents constrained ordination; thus, environmental variables directly influence the ordination process (Legendre and Legendre 2012). The environmental variables measured for this study were altitude, atmospheric temperature, humidity, and soil surface temperature. We included these variables in the initial model including the main factors (i.e., season and location) and then selected the best-fit model to perform redundancy analysis with this final model. We used a Hellinger transformation on the species data to minimise the weight of rare species (Legendre and Gallagher 2001).

We used rarefaction and extrapolation to standardise uneven sampling efforts and to estimate species richness among seasons and locations. Rarefaction and extrapolation better characterise species richness than conventional individual-based rarefaction because it compares assemblages at a minimum coverage level in contrast with a minimum number of individuals (Chao et al. 2014a, 2014b). By doubling the reference sample size, extrapolation better predicts the presence of rare species (Budka et al. 2019). We visualised estimated species richness using the "ggiNEXT" function in R (Hsieh et al. 2016).

We used a generalised linear model, with residuals following a negative binomial distribution to analyse differences in abundance of adult beetles under different seasons and locations. We used Tukey's honestly significant difference test for multiple comparisons of species richness and abundance when results from the generalised linear modelling were significant.

We used indicator species analysis to identify species associated with specific seasons and locations (Dufrêne and Legendre 1997). Indicator species analysis is an analytical method used to evaluate the statistical significance of relationships between species occurrence or abundance and any defined group (De Cáceres et al. 2010). We generated the "IndVal" function using 4999 permutations and calculated $P$-values for each species to ensure the results were different from random $(\alpha=0.05)$.

All analyses, except for permutational multivariate analysis of variance, were conducted using R, version 3.6.3 (R Core Team 2020), using the "Vegan" (Oksanen et al. 2019), "MASS" (Venables and Ripley 2002), "multcomp" (Hothorn et al. 2008), "iNEXT" (Hsieh et al. 2016), and “indicspecies” packages (De Cáceres and Legendre 2009). 


\section{General results}

\section{Results}

A total of 5820 arthropods, representing six classes, were collected over four seasons (Supplementary material, Table S1). These arthropods included 4125 insects representing 11 orders (Supplementary material, Table S2). Among these insects, 1422 adult beetles representing 24 families and a minimum of 141 species were collected, with 73 singletons and 18 doubletons (Table 1, Supplementary material, Table S3). The minute hooded beetle, Lewisium japonicum (Corylophidae), was the dominant species collected among the beetles, with 376 individuals, and accounted for $26 \%$ of the total beetle catch. Two aleocharine rove beetles (Staphylinidae), Atheta koreana and A. pasniki, were the second- and the third-most common species, with 80 and 70 individuals, respectively (Table 1).

\section{Beetle assemblage composition}

Results of the permutational multivariate analysis of variance showed that adult beetleassemblage composition differed significantly in terms of season (pseudo- $F=2.35, P=0.001$ ) and location (pseudo- $F=1.50, P=0.018$ ), but the results showed no interaction between season and location (pseudo- $F=0.94, P=0.676$; Table 2). Pairwise comparisons showed that beetle assemblage composition differed among seasons, except between spring and winter. In terms of location, assemblage composition was similar between $\mathrm{Na}$ and Suk, whereas the assemblage composition in Jol differed significantly from that of $\mathrm{Na}$ and Suk (Table 2).

According to the results of the redundancy analysis, the final model revealed significant differences across seasons $(F=2.09, P=0.005)$ and locations $(F=1.35, P=0.035)$. Overall assemblage structure was significantly correlated with soil surface temperature $(F=2.74$, $P=0.005)$ and atmospheric temperature $(F=2.16, P=0.005)$ along axis 1 (Table 3; Fig. 4). The model explained $31.6 \%$ of the total variance, with axes 1 and 2 accounting for $10.4 \%$ and $5.7 \%$ of the variance, respectively (Fig. 4). The permutational multivariate analysis of variance results were strongly supported by the redundancy analysis ordination, indicating that the assemblage composition differed among seasons except for a slight overlap during spring and winter. Also, the ellipse for summer assemblages was the largest, and the ellipse for winter assemblages was the smallest, suggesting that assemblages in summer and winter were the most heterogeneous and homogeneous, respectively (Fig. 4a). Two temperature variables seemed to drive seasonal differences of beetle assemblage structure along axis 1 (Fig. 4a). In terms of location, the redundancy analysis ordination showed that the assemblage composition was similar between $\mathrm{Na}$ and Suk (Fig. 4b). Furthermore, the ellipse for Jol assemblages was smallest, suggesting that the assemblages in Jol were more homogeneous than those in $\mathrm{Na}$ and Suk. Although it was not significant, altitude and humidity tended to drive regional variations of beetle assemblages along axis 2 (Fig. $4 \mathrm{~b}$ ).

\section{Species richness and number of individuals}

Estimated species richness of adult beetles was highest in spring and lowest in summer at $67.8 \%$ and $96.3 \%$ sample coverages, respectively, based on extrapolated curves (Fig. 5a). Estimated species richness was similar between fall and winter, both at $93.4 \%$ sample coverages, showing higher species richness than that in summer. However, species richness in fall and winter did not differ significantly from that in spring (Fig. 5a). Estimated species richness did not differ among locations, at $90.8 \%, 95.0 \%$, and $92.8 \%$ sample coverages for Jol, Na, and Suk, respectively (Fig. 5b). However, Suk tended to be highest in species richness, whereas Jol tended to be lowest (Fig. 5b). 
Table 1. List of adult beetles with the number of individuals collected in four seasons in Jeongseon-gun, Gangwon-do, Korea, using a sifting method followed by Berlese funnel extraction.

\begin{tabular}{|c|c|c|c|c|c|c|c|}
\hline \multirow{2}{*}{\multicolumn{2}{|c|}{ Scientific name }} & \multirow[b]{2}{*}{ Feeding guild } & \multicolumn{4}{|c|}{ Month } & \multirow[b]{2}{*}{ Total individuals } \\
\hline & & & Mar & Aug & Oct & Dec & \\
\hline \multicolumn{8}{|c|}{ Anthicidae } \\
\hline 1 & Macratria sp.1 & Unknown & & & & 1 & 1 \\
\hline \multicolumn{8}{|c|}{ Carabidae } \\
\hline 2 & Bembidion scopulinum Kirby & Predator & 1 & & & & 1 \\
\hline 3 & Bembidion sp.1 & Predator & & 2 & & & 2 \\
\hline 4 & Bradycellus sp.1 & Omnivore & & & 1 & & 1 \\
\hline 5 & Colpodes sp.1 & Predator & & 1 & & & 1 \\
\hline 6 & Nebria sp.1 & Predator & & 2 & & & 2 \\
\hline 7 & Perigona nigriceps Dejean & Unknown & & 5 & 2 & & 7 \\
\hline 8 & Pterostichus sp.1 & Unknown & & 2 & & & 2 \\
\hline 9 & Trechus sp.1 & Unknown & & 1 & & & 1 \\
\hline 10 & Trichotichnus congruus (Motschulsky) & Omnivore & 1 & & & & 1 \\
\hline 11 & Trichotichnus gerni Noonan & Omnivore & & & 2 & & 2 \\
\hline 12 & Trichotichnus sp.1 & Omnivore & & & 1 & & 1 \\
\hline \multicolumn{8}{|c|}{ Chrysomelidae } \\
\hline 13 & Agelasa nigriceps Motschulsky & Herbivore & & & 1 & 6 & 7 \\
\hline 14 & Altica sp.1 & Herbivore & & & & 1 & 1 \\
\hline 15 & Cassida fuscorufa Motschulsky & Herbivore & & & & 1 & 1 \\
\hline 16 & Cassida pallidicollis Boheman & Herbivore & & & & 1 & 1 \\
\hline 17 & Chrysochus chinensis Baly & Herbivore & & & & 1 & 1 \\
\hline \multicolumn{8}{|c|}{ Ciidae } \\
\hline 18 & Cis sp. 1 & Fungivore & 1 & & & & 1 \\
\hline \multicolumn{8}{|c|}{ Coccinellidae } \\
\hline 19 & Pseudoscymnus hareja Weise & Predator & 1 & & & & 1 \\
\hline \multicolumn{8}{|c|}{ Corylophidae } \\
\hline 20 & Lewisium japonicum Matthews & Fungivore & 36 & 119 & 88 & 133 & 376 \\
\hline \multicolumn{8}{|c|}{ Cryptophagidae } \\
\hline 21 & Atomaria lewisi Reitter & Fungivore & & 1 & & 8 & 9 \\
\hline 22 & Atomaria sp.1 & Fungivore & & & 2 & & 2 \\
\hline 23 & Caenoscelis sibirica Reitter & Fungivore & & & & 2 & 2 \\
\hline 24 & Cryptophagus sp.1 & Fungivore & 1 & 1 & 1 & & 3 \\
\hline 25 & Cryptophagus sp.2 & Fungivore & 1 & 1 & 1 & & 3 \\
\hline 26 & Micrambe sp.1 & Herbivore & 2 & & & & 2 \\
\hline \multicolumn{8}{|c|}{ Curculionidae } \\
\hline 27 & Acallinus tuberculatus Morimoto & Herbivore & & & & 1 & 1 \\
\hline
\end{tabular}


Table 1. (Continued)

\begin{tabular}{|c|c|c|c|c|c|c|c|}
\hline & & \multirow[b]{2}{*}{ Feeding guild } & \multicolumn{4}{|c|}{ Month } & \multirow[b]{2}{*}{ Total individuals } \\
\hline \multicolumn{2}{|c|}{ Scientific name } & & Mar & Aug & Oct & Dec & \\
\hline 28 & Acicnemis luteomaculata (Morimoto) & Herbivore & & & & 1 & 1 \\
\hline 29 & Catabonops monachus Roelofs & Herbivore & 1 & & & & 1 \\
\hline 30 & Catabonops sp.1 & Herbivore & & & & 1 & 1 \\
\hline 31 & Ceutorhynchus sinicus (Voss) & Herbivore & 1 & & & & 1 \\
\hline 32 & Cyrtepistomus castaneus (Roelofs) & Herbivore & & & & 1 & 1 \\
\hline 33 & Dryocoetes pini Niisima & Herbivore & & 1 & & & 1 \\
\hline 34 & Larinus sp.1 & Herbivore & 1 & & & & 1 \\
\hline 35 & Lepidepistomodes fumosus (Faust) & Herbivore & & & & 1 & 1 \\
\hline 36 & Myosides chejuensis Morimoto and Lee & Herbivore & & & & 5 & 5 \\
\hline 37 & Nothomyllocerus griseus (Roelofs) & Herbivore & & 2 & & & 2 \\
\hline 38 & Nothomyllocerus illitus (Reitter) & Herbivore & & & & 1 & 1 \\
\hline 39 & Polygraphus sp.1 & Herbivore & & & & 1 & 1 \\
\hline 40 & Ptochidius sp.1 & Herbivore & & 1 & & & 1 \\
\hline 41 & Ptochidius tessellatus Motschulsky & Herbivore & 1 & & & 2 & 3 \\
\hline 42 & Scepticus sp.1 & Herbivore & 1 & & & & 1 \\
\hline 43 & Trachodes subfasciatus Voss & Herbivore & 1 & & & & 1 \\
\hline \multicolumn{8}{|c|}{ Discolomatidae } \\
\hline 44 & Aphanocephalus hemisphericus Wollaston & Fungivore & & & 1 & 1 & 2 \\
\hline \multicolumn{8}{|c|}{ Elateridae } \\
\hline 45 & Ampedus sp.1 & Unknown & & & 1 & & 1 \\
\hline \multicolumn{8}{|c|}{ Endomychidae } \\
\hline 46 & Ancylopus pictus asiaticus Strohecker & Fungivore & & 1 & & & 1 \\
\hline 47 & Mycetina stackelbergi Kryzhanowskij & Fungivore & & & 1 & & 1 \\
\hline \multicolumn{8}{|c|}{ Hydraenidae } \\
\hline 48 & Hydraena riparia Kugelann & Omnivore & & & 1 & & 1 \\
\hline \multicolumn{8}{|c|}{ Hydrophilidae } \\
\hline 49 & Cercyon sp.1 & Saprophage & & 3 & 12 & & 15 \\
\hline 50 & Cercyon sp.2 & Saprophage & & 1 & & & 1 \\
\hline 51 & Cercyon sp.3 & Saprophage & & 2 & & & 2 \\
\hline \multicolumn{8}{|c|}{ Latridiidae } \\
\hline 52 & Corticaria ornata Reitter & Fungivore & & & 1 & 2 & 3 \\
\hline 53 & Corticarina lutea Rücker & Fungivore & & & 3 & & 3 \\
\hline 54 & Dienerella costulata (Reitter) & Fungivore & & & 1 & & 1 \\
\hline 55 & Stephostethus chinensis (Reitter) & Fungivore & 2 & & 2 & & 4 \\
\hline \multicolumn{8}{|c|}{ Leiodidae } \\
\hline 56 & Agathidium badium Erichson & Fungivore & & & & 1 & 1 \\
\hline
\end{tabular}


Table 1. (Continued)

\begin{tabular}{|c|c|c|c|c|c|c|c|}
\hline & & \multirow[b]{2}{*}{ Feeding guild } & \multicolumn{4}{|c|}{ Month } & \multirow[b]{2}{*}{ Total individuals } \\
\hline \multicolumn{2}{|c|}{ Scientific name } & & Mar & Aug & Oct & Dec & \\
\hline 57 & Agathidium merkli Angelini & Fungivore & & & & 1 & 1 \\
\hline 58 & Agathidium sp.1 & Fungivore & & & 1 & & 1 \\
\hline 59 & Agathidium sp.2 & Fungivore & & & 4 & & 4 \\
\hline 60 & Leiodidae sp.1 & Fungivore & & & & 8 & 8 \\
\hline 61 & Leiodidae sp.2 & Fungivore & & & & 1 & 1 \\
\hline 62 & Pseudcolenis hilleri Reitter & Fungivore & & & & 3 & 3 \\
\hline 63 & Typhlocolenis sp.1 & Fungivore & & & 1 & & 1 \\
\hline 64 & Typhlocolenis sp.2 & Fungivore & & & 2 & 2 & 4 \\
\hline \multicolumn{8}{|c|}{ Lucanidae } \\
\hline 65 & Platycerus hongwonpyoi Imura \& Choe & Herbivore & & & & 1 & 1 \\
\hline \multicolumn{8}{|c|}{ Melandryidae } \\
\hline 66 & Lederina sp.1 & Xylophage & 1 & & & & 1 \\
\hline 67 & Lederina koreana Choi, Park, Kim and Park & Xylophage & & & 3 & & 3 \\
\hline \multicolumn{8}{|c|}{ Nitidulidae } \\
\hline 68 & Epuraea oblonga (Herbst) & Omnivore & & & & 1 & 1 \\
\hline 69 & Epuraea hisamatsui Nakane & Omnivore & & 1 & & 1 & 2 \\
\hline 70 & Meligethes flavicollis Reitter & Unknown & & & 2 & 2 & 4 \\
\hline 71 & Pallodes sp.1 & Unknown & & & 1 & & 1 \\
\hline 72 & Prometopia unidentata Hisamatsu & Unknown & & & & 1 & 1 \\
\hline \multicolumn{8}{|c|}{ Ptiliidae } \\
\hline 73 & Acrotrichis lewisi (Matthews) & Fungivore & & & & 5 & 5 \\
\hline 74 & Acrotrichis sp.1 & Fungivore & & 4 & 1 & 15 & 20 \\
\hline 75 & Baeocrara sp.1 & Fungivore & & & 1 & 64 & 65 \\
\hline 76 & Ptinella alisonae Darby & Saprophage & & & & 7 & 7 \\
\hline 77 & Ptinella sp.1 & Saprophage & & 16 & 52 & & 68 \\
\hline \multicolumn{8}{|c|}{ Rhynchitidae } \\
\hline 78 & Aspidobyctiscus lacunipennis (Jekel) & Herbivore & & & & 1 & 1 \\
\hline \multicolumn{8}{|c|}{ Silvanidae } \\
\hline 79 & Silvanoprus angusticollis (Reitter) & Fungivore & & & & 1 & 1 \\
\hline 80 & 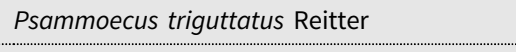 & Unknown & & & & 1 & 1 \\
\hline \multicolumn{8}{|c|}{ Sphindidae } \\
\hline 81 & Aspidiphorus japonicus Reitter & Fungivore & & 1 & 1 & 1 & 3 \\
\hline 82 & Aspidiphorus sakaii Sasaji & Fungivore & & & 2 & & 2 \\
\hline \multicolumn{8}{|c|}{ Staphylinidae } \\
\hline \multicolumn{8}{|c|}{ Aleocharinae } \\
\hline 83 & Aleochara sp.1 & Predator & & & & 5 & 5 \\
\hline
\end{tabular}


Table 1. (Continued)

\begin{tabular}{|c|c|c|c|c|c|c|c|}
\hline & & \multirow[b]{2}{*}{ Feeding guild } & \multicolumn{4}{|c|}{ Month } & \multirow[b]{2}{*}{ Total individuals } \\
\hline \multicolumn{2}{|c|}{ Scientific name } & & Mar & Aug & Oct & Dec & \\
\hline 84 & Aleochara sp.2 & Predator & & & & 30 & 30 \\
\hline 85 & Aleochara sp.3 & Predator & & & & 1 & 1 \\
\hline 86 & Atheta koreana Bernhauer & Predator & & & & 80 & 80 \\
\hline 87 & Atheta pasniki Lee \& Ahn & Predator & & & & 70 & 70 \\
\hline 88 & Atheta spinula (Sawada) & Predator & & & & 1 & 1 \\
\hline 89 & Atheta sp.1 & Predator & 1 & & 5 & 11 & 17 \\
\hline 90 & Atheta sp.2 & Predator & & & & 1 & 1 \\
\hline 91 & Atheta sp.3 & Predator & & & & 31 & 31 \\
\hline 92 & Homalota sauteri Bernhauer & Predator & & & & 1 & 1 \\
\hline 93 & Leptusa sp.1 & Predator & & & 1 & & 1 \\
\hline 94 & Paracyusa sp.1 & Predator & 1 & & & & 1 \\
\hline 95 & Aleocharinae spp. & Predator & 2 & 53 & 123 & 1 & 179 \\
\hline \multicolumn{8}{|c|}{ Omaliinae } \\
\hline 96 & Geodromicus sp.1 & Unknown & & & & 1 & 1 \\
\hline 97 & Lesteva sp.1 & Unknown & 1 & & & & 1 \\
\hline 98 & Omalium sp.1 & Unknown & & & 1 & & 1 \\
\hline 99 & Psephidonus sp.1 & Unknown & & & 3 & & 3 \\
\hline \multicolumn{8}{|c|}{ Osoriinae } \\
\hline 100 & Osorius taurus Sharp & Saprophage & & & 11 & & 11 \\
\hline 101 & Osorius sp.1 & Saprophage & & 9 & & & 9 \\
\hline \multicolumn{8}{|c|}{ Oxytelinae } \\
\hline 102 & Oxytelus sp.1 & Predator & 1 & & 2 & & 3 \\
\hline 103 & Oxytelinae spp. & Unknown & & 37 & & & 37 \\
\hline \multicolumn{8}{|c|}{ Paederinae } \\
\hline 104 & Achenomorphus sp.1 & Predator & & & 2 & & 2 \\
\hline 105 & Astenus sp.1 & Predator & & & 1 & & 1 \\
\hline 106 & Leptacinus sp.1 & Predator & & & 2 & & 2 \\
\hline 107 & Medon spadiceus Sharp & Predator & 1 & & & & 1 \\
\hline 108 & Nazeris rutilicorpus Cho & Predator & 1 & & & & 1 \\
\hline 109 & Paederinae spp. & Predator & & 43 & & & 43 \\
\hline \multicolumn{8}{|c|}{ Piestinae } \\
\hline 110 & Siagonium sp.1 & Fungivore & & & 1 & & 1 \\
\hline \multicolumn{8}{|c|}{ Proteininae } \\
\hline & Megarthrus corticalis Sharp & Fungivore & & & & 2 & 2 \\
\hline 112 & Megarthrus japonicus Sharp & Fungivore & & & & 2 & 2 \\
\hline 113 & Megarthrus sawadai Cuccodoro & Fungivore & & & & 7 & 7 \\
\hline
\end{tabular}


Table 1. (Continued)

\begin{tabular}{|c|c|c|c|c|c|c|c|}
\hline \multirow{2}{*}{\multicolumn{2}{|c|}{ Scientific name }} & \multirow{3}{*}{$\begin{array}{l}\text { Feeding guild } \\
\text { Fungivore }\end{array}$} & \multicolumn{4}{|c|}{ Month } & \multirow{3}{*}{$\frac{\text { Total individuals }}{4}$} \\
\hline & & & \multirow{2}{*}{$\begin{array}{c}\text { Mar } \\
1\end{array}$} & \multirow[t]{2}{*}{ Aug } & \multirow{2}{*}{$\frac{\text { Oct }}{3}$} & \multirow[t]{2}{*}{ Dec } & \\
\hline 114 & Megarthrus sp.1 & & & & & & \\
\hline 115 & Megarthrus sp.2 & Fungivore & & & 1 & & 1 \\
\hline \multicolumn{8}{|c|}{ Pselaphinae } \\
\hline 116 & Batrisodes baejeongdoki Nomura \& Lee & Predator & & 5 & 1 & & 6 \\
\hline 117 & Bryaxis leechanyoungi Nomura \& Lee & Predator & & 16 & 11 & 2 & 29 \\
\hline 118 & Bryaxis kimjongkuki Nomura \& Lee & Predator & & & 2 & & 2 \\
\hline 119 & Euplectus domefactus (Kurbatov) & Predator & & & 1 & & 1 \\
\hline 120 & Pselaphogenius cornurus (Nomura \& Lee) & Predator & & & & 1 & 1 \\
\hline 121 & Pselaphus striatus Löbl & Predator & & & 1 & & 1 \\
\hline \multicolumn{8}{|c|}{ Scaphidiinae } \\
\hline 122 & Baeocera sp.1 & Fungivore & & & & 1 & 1 \\
\hline \multicolumn{8}{|c|}{ Scydmaeninae } \\
\hline 123 & Euconnus sp.1 & Predator & & & 1 & 1 & 2 \\
\hline \multicolumn{8}{|c|}{ Staphylininae } \\
\hline 124 & Gabrius sp.1 & Predator & 1 & & & & 1 \\
\hline 125 & Philonthus sp.1 & Predator & & & 5 & 2 & 7 \\
\hline 126 & Philonthus sp.2 & Predator & & & & 7 & 7 \\
\hline 127 & Staphylininae spp. & Predator & & 6 & & & 6 \\
\hline \multicolumn{8}{|c|}{ Steninae } \\
\hline 128 & Stenus sp.1 & Predator & & 2 & 8 & 1 & 11 \\
\hline 129 & Stenus sp.2 & Predator & & 6 & & & 6 \\
\hline \multicolumn{8}{|c|}{ Tachyporinae } \\
\hline 130 & Sepedophilus germanus Sharp & Fungivore & 4 & 24 & & & 28 \\
\hline 131 & Sepedophilus testaceus (Fabricius) & Fungivore & & & & 6 & 6 \\
\hline 132 & Sepedophilus sp.1 & Fungivore & & & 30 & 6 & 36 \\
\hline 133 & Derops coreanus (Watanabe) & Unknown & & 10 & & & 10 \\
\hline 134 & Ischnosoma sp.1 & Predator & & & & 1 & 1 \\
\hline 135 & Nitidotachinus sp.1 & Predator & & & 1 & & 1 \\
\hline 136 & Tachinus sp.1 & Predator & & & & 1 & 1 \\
\hline 137 & Tachyporinae sp.1 & Unknown & & 1 & & & 1 \\
\hline \multicolumn{8}{|c|}{ Tenebrionidae } \\
\hline 138 & Tetraphyllus sp.1 & Saprophage & 1 & & & & 1 \\
\hline 139 & Uloma bonzica Marseul & Saprophage & & 1 & & & 1 \\
\hline 140 & Misolampidius tentyrioides Solsky & Saprophage & & 1 & & & 1 \\
\hline 141 & Stenophanes mesostena (Solsky) & Saprophage & & 1 & & & 1 \\
\hline \multicolumn{3}{|c|}{ Total 24 families, 100 genera, and minimum 141 species } & 68 & 383 & 410 & 561 & 1422 \\
\hline
\end{tabular}


Table 2. Results of permutational multivariate analysis of variance testing for adult beetle species richness by season and location.

\begin{tabular}{ccccccc}
\hline & $d f$ & SS & MS & Pseudo- $F$ & $P($ PERM $)$ & Pairwise comparisons \\
\hline Season & 3 & 19785.0 & 6594.8 & 2.35 & $\mathbf{0 . 0 0 1}$ & (Spring = Winter) $\neq$ Summer $\neq$ Fall \\
\hline Location & 2 & 8426.4 & 4213.2 & 1.50 & $\mathbf{0 . 0 1 8}$ & Jol $\neq(\mathrm{Na}=$ Suk $)$ \\
\hline Se $\times$ Lo & 6 & 15819 & 2636.4 & 0.94 & 0.676 & Season and location, no interaction \\
\hline
\end{tabular}

Significant differences $(P<0.05)$ are highlighted in bold.

Abbreviations: $d f$, degrees of freedom; Lo, location; MS, mean squares; PERM, permutational multivariate analysis of variance; Se, season; SS, sum of squares.

Table 3. Results of redundancy analysis testing for adult beetle assemblages by factors.

\begin{tabular}{lcccc}
\hline & Df & AIC & $F$ & $\operatorname{Pr}(>\mathrm{F})$ \\
\hline S.Temp & 1 & -13.998 & 2.7446 & $\mathbf{0 . 0 0 5}$ \\
\hline Season & 3 & -13.678 & 2.0937 & $\mathbf{0 . 0 0 5}$ \\
\hline Temp & 1 & -13.428 & 2.1663 & $\mathbf{0 . 0 0 5}$ \\
\hline Hum & 1 & -12.410 & 1.1599 & 0.190 \\
\hline Location & 2 & -12.055 & 1.3536 & $\mathbf{0 . 0 3 5}$ \\
\hline Alt & 1 & -11.995 & 0.7584 & 0.03 \\
\hline
\end{tabular}

Significant differences $(P<0.05)$ are noted in bold.

Abbreviations: $d f$, degrees of freedom; AIC, Akaike information criteria; S.Temp, soil surface temperature; Temp, atmospheric temperature; Hum, humidity; Alt, altitude.

Adult beetle abundance differed significantly among seasons (deviance $=50.7, P<0.001$ ), with the lowest abundance occurring in spring (Fig. 6a). However, beetle abundance did not differ significantly among locations (deviance $=37.8, P=0.108$; Fig. $6 \mathrm{~b}$ ).

\section{Indicator species analysis}

Nine and three species were identified as significant indicators of season and location, respectively. All indicators of season were members of Staphylinidae, except for the featherwing beetle, Ptinella sp.1 (Ptiliidae). In terms of season, Aleocharinae spp. showed the highest indicator value in fall (IndVal $=0.830$ ), followed by Sepedophilus germanus (Sharp) in summer (IndVal = 0.782). Philonthus sp. 1 was the only indicator in winter $($ IndVal $=0.579$ ). No indicator species were found in spring (Table 4). Based on location, Perigona nigriceps Dejean showed the highest indicator value in Jol (IndVal = 0.612), followed by Cryptophagus sp. 1 and Meligethes flavicollis Reitter, showing the same indicator value in $\mathrm{Na}$ (IndVal =0.577; Table 4).

\section{Discussion}

\section{Seasonal variations of adult beetle assemblage structure}

The present study clearly showed that adult beetle assemblages differed among seasons, except between spring and winter, which partly overlapped. This similarity in assemblage structure between spring and winter is interesting. It is plausible that a large number of adult species overwintering under leaf litter emerged and were collected in spring, thereby displaying a similar assemblage structure between spring and winter (Burgess 1981; Bale and Hayward 2010). In addition, the dissimilarity of beetle assemblages between spring, summer, 

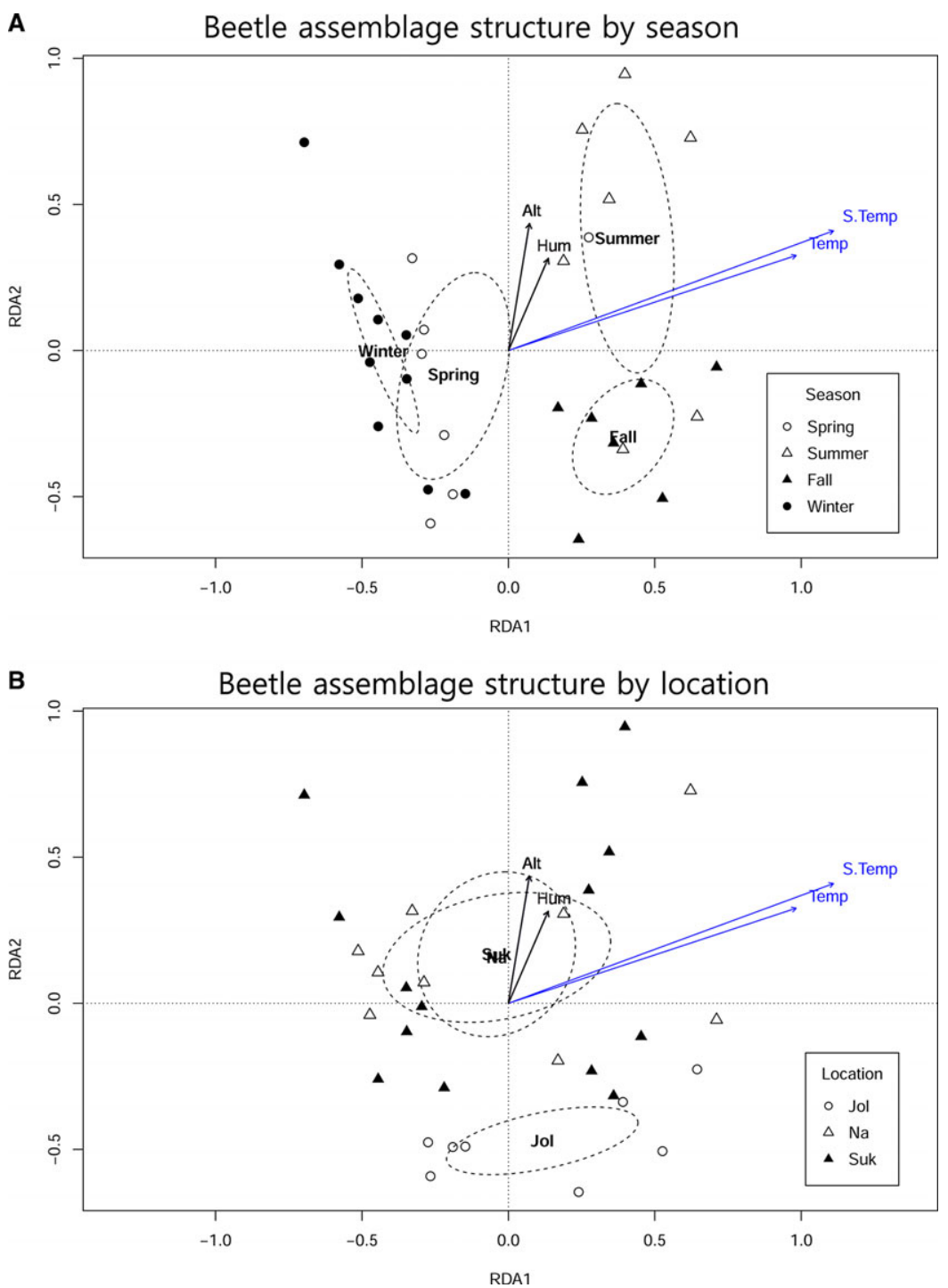

Fig. 4. Redundancy analysis (RDA) ordination of adult beetle assemblages by $\mathbf{A}$, season and $\mathbf{B}$, location. The model explained $31.6 \%$ of the total variance, and axis 1 and axis 2 explained $10.3 \%$ and $5.5 \%$ of the total variance, respectively. Arrows indicate environmental variables, with significant factors coloured blue. Abbreviations: Alt, altitude; Hum, humidity; S.Temp, soil surface temperature; Temp, atmospheric temperature.

and fall emphasises that each species has a different developmental plan, thereby emerging as adults in a different season. The distinct assemblage structure among seasons suggests the importance of sampling in different seasons to understand the total diversity of litter-dwelling beetles. A transition from dry to rainy seasons in summer may have produced different adult beetle assemblage structures due to environmental changes (Anu et al. 2009). Our study also revealed that the summer beetle assemblage was the most heterogeneous. Because of the high temperature in summer, activity and dispersibility of insects increase (Mellanby 1939; Taylor 1963), leading to structurally diverse summer communities. Winter assemblages were the most homogeneous, possibly due to low insect dispersibility and immobility or diapause (Bertram 1935; Mellanby 1939; Bale and Hayward 2010). 

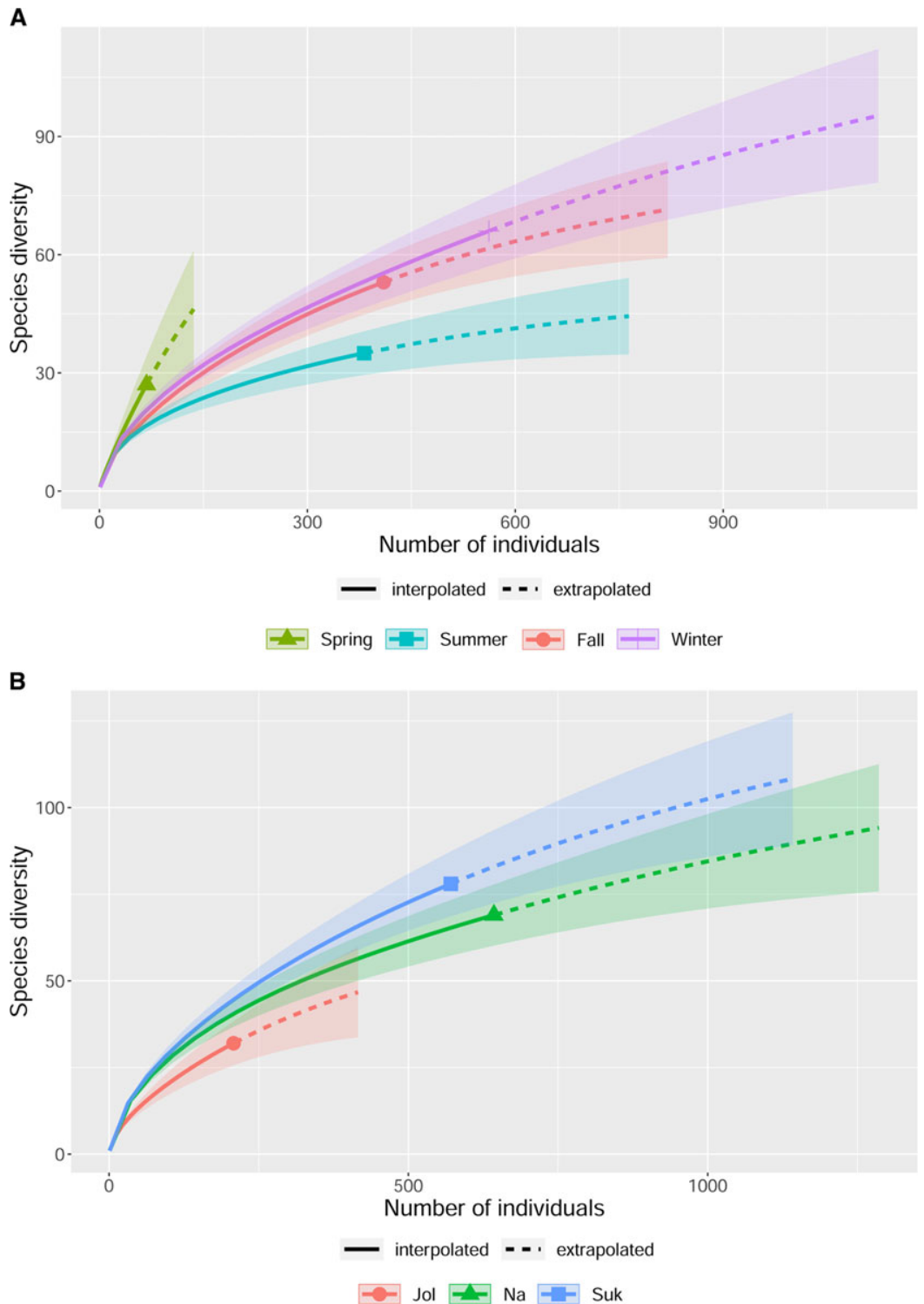

Fig. 5. Estimated species richness for $\mathbf{A}$, season and $\mathbf{B}$, location. Solid lines indicate interpolation, and dotted lines indicate extrapolation. Shaded areas indicate $95 \%$ confidence intervals.

\section{Seasonal changes in species richness and abundance}

Previous studies have shown the highest species richness and abundance of adult beetles occurred in either summer or fall in Korea (Byun et al. 2009a, 2009b, 2010; Park et al. 2011; Lim et al. 2011, 2012). Contrary to our prediction, the results of the present study showed that species richness was lowest in summer and that richness in winter was as high as that in spring and fall. In addition, abundance in winter was similar to values found for summer and fall, suggesting that winter sampling using a sifting method was highly effective for sampling adult beetles. Some species found in litter samples in winter may not be directly related to the soil and leaf-litter environment, as they use this habitat only for overwintering. The lowest abundance of adult beetles in spring may be attributed to dried leaf litter and soils that may 

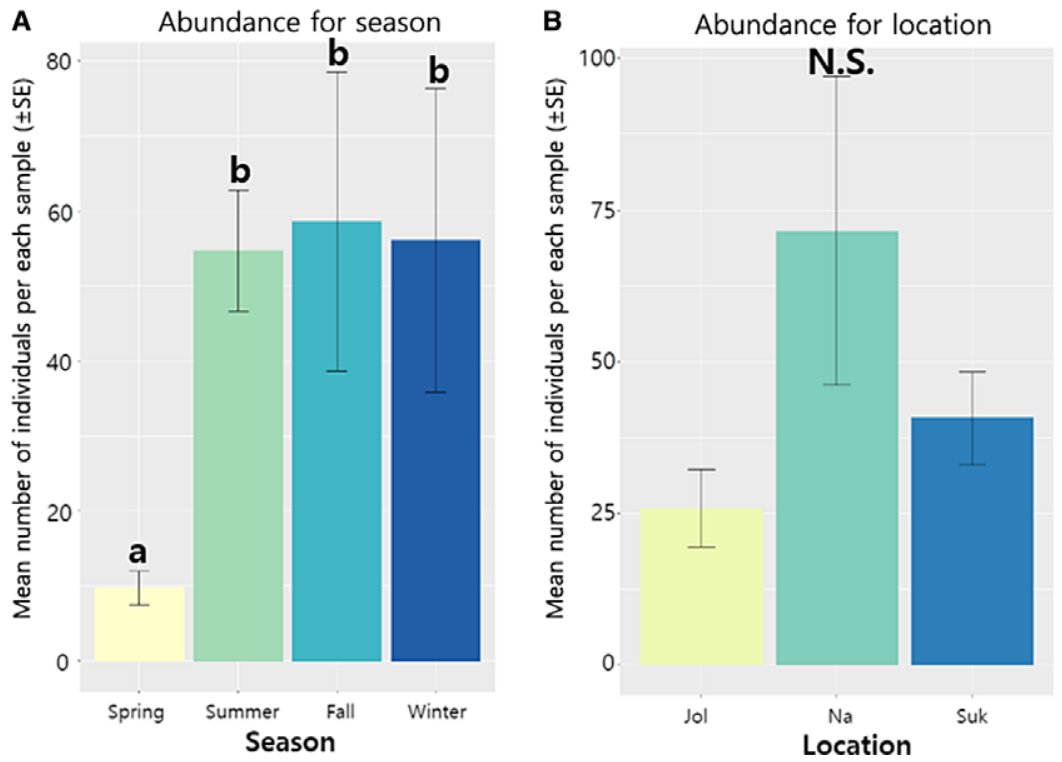

Fig. 6. Adult beetle abundance for A, season and B, location. Error bars represent the standard errors (SE) for each location. Different lowercase letters above error bars show significant post hoc results. N.S., nonsignificant post hoc result (Tukey's honestly significant difference test, $P<0.05)$.

Table 4. Significant indicator species of adult beetles for season and location.

\begin{tabular}{|c|c|c|c|c|c|}
\hline Group & Family & Species & Feeding guild & IndVal* & $P$ \\
\hline \multicolumn{6}{|l|}{ Season } \\
\hline Summer & Staphylinidae & Sepedophilus germanus & Fungivore & 0.782 & 0.0020 \\
\hline Summer & Staphylinidae & Oxytelinae spp. & Unknown & 0.756 & 0.0032 \\
\hline Summer & Staphylinidae & Paederinae spp. & Predator & 0.756 & 0.0032 \\
\hline Summer & Staphylinidae & Derops coreanus & Fungivore & 0.655 & 0.0228 \\
\hline Fall & Staphylinidae & Aleocharinae spp. & Predator & 0.830 & 0.0056 \\
\hline Fall & Staphylinidae & Osorius taurus & Saprophage & 0.756 & 0.0040 \\
\hline Fall & Ptiliidae & Ptinella sp.1 & Saprophage & 0.661 & 0.0402 \\
\hline Fall & Staphylinidae & Philonthus sp.1 & Predator & 0.579 & 0.0362 \\
\hline Winter & Staphylinidae & Aleochara sp.1 & Predator & 0.632 & 0.0116 \\
\hline \multicolumn{6}{|l|}{ Location } \\
\hline Jol & Carabidae & Perigona nigriceps & Unknown & 0.612 & 0.0130 \\
\hline $\mathrm{Na}$ & Cryptophagidae & Cryptophagus sp.1 & Fungivore & 0.577 & 0.0308 \\
\hline $\mathrm{Na}$ & Nitidulidae & Meligethes flavicollis & Omnivore & 0.577 & 0.0298 \\
\hline
\end{tabular}

*Indicator value

$P$-values were calculated after 4999 permutations.

cause beetles to move into more humid habitats, such as under logs or under bark (Janzen and Schoener 1968; Pinheiro et al. 2002). Most biodiversity studies in temperate forests use sweeping, light traps, Malaise traps, window, or pitfall traps to sample arthropods (Gadagkar et al. 1990; Recher et al. 1996; Hébert et al. 2000; Kai and Corlett 2002; Work et al. 2002; Park and 
Cho 2007; Santos et al. 2007; Vasconcellos et al. 2010; Park et al. 2011; Lim et al. 2012; Dadmal and Khadakkar 2014; Saji and Al Dhaheri 2014; Kim and Kwon 2016; Wardhaugh et al. 2018). Because of this, it was difficult to directly compare the results for arthropod diversity and abundance of the present study with those of other studies due to the different sampling methods used by prior studies to collect biological data in the winter.

Only a few studies have used a combined sifting-Berlese funnel method to investigate insect biodiversity (Carlton and Robison 1998; Ferro et al. 2012a, 2012b). For example, Carlton and Robison (1998) observed the highest abundance of litter-dwelling beetles in spring and fall and the lowest abundance in early summer and winter. Ferro et al. (2012b) reported that both species richness and abundance of adult beetles were higher in spring than in fall. Differences between the present study and other studies were: (1) the types of material used during sifting, as they also sifted rotten deadwood together with leaf litters and soils; (2) the operation time for the funnel extraction (one week in the present study versus two days in the earlier studies); and (3) environmental factors, such as temperature, humidity, and rainfall in each sampling region.

\section{Effects of environmental factors on beetle assemblage}

Temperature affects insect abundance and assemblages (Wagner et al. 1985; Gilbert and Raworth 1996; Régnière et al. 2012). In our study, assemblage structures of adult beetles were affected by both soil surface and atmospheric temperatures, supporting previous findings that litter-dwelling beetles were sensitive to both soil surface temperature (Robinson et al. 2018) and atmospheric temperature (Ruggiero et al. 2009). Other factors, such as humidity and soil $\mathrm{pH}$, showed no significant effects on variation in the assemblage structure.

Precipitation and humidity also significantly affect insect communities (Robinson and Robinson 1970; Smythe 1973; Wolda 1978a, 1978b; Rees 1983; Frith and Frith 1990; Pinheiro et al. 2002). However, in the present study, beetle assemblages were not affected by humidity. Characteristics of litter samples in our study areas were similar to each other, with wet soils and deciduous leaf litters. Soil $\mathrm{pH}$ in our study areas also did not affect beetle assemblage structure, supporting the results of Cameron and Leather (2012), who suggested that soil pH was unrelated to insect and carabid abundance. In our study areas, soil $\mathrm{pH}$ ranged between 6.7 and 7.0, showing no significant difference among seasons or locations. Additional environmental factors, such as vegetation, soil composition, and presence of deadwood, are better predictors of the structure of litter-dwelling arthropods and their environmental relationships (Ulyshen and Hanula 2009; Ashford et al. 2013).

\section{Regional variations in adult beetle diversity and community structure}

Both $\mathrm{Na}$ and Suk showed similar adult beetle assemblage structure despite these sites being the farthest apart. Beetle assemblages in $\mathrm{Na}$ and Suk were similarly heterogeneous, whereas those in Jol showed the maximum homogeneity. The lack of differences in annual average temperatures and soil surface temperatures at $\mathrm{Na}$ and Suk may help explain the similar beetle assemblage structures in these areas. Although we did not investigate plant species as an environmental variable, the different species composition of plants at each sampling area may have influenced the beetle assemblage composition (Andow 1991; Koricheva et al. 2000; Scherber et al. 2006).

The Jol site had lower species richness and abundance of adult beetles compared to the other sites. Since the sample area at Jol was narrower compared to those at $\mathrm{Na}$ and Suk, the least richness and abundance at Jol were expected. Conversely, $\mathrm{Na}$ tended to show high species richness and abundance of beetles. The average elevation at $\mathrm{Na}(782 \mathrm{~m})$ was higher than at Jol $(384 \mathrm{~m})$ and Suk $(450 \mathrm{~m})$, and this difference likely affected the diversity and assemblage structure. 
Previous studies have reported changes in diversity and assemblages along an elevational gradient (Lawton et al. 1987; Wolda 1987; Olson 1994; Sánchez-Reyes et al. 2014).

\section{Advantages of sampling winter assemblages using a sifting method}

Sampling litter-dwelling arthropods in winter is challenging because many of them enter diapause to survive the cold. Therefore, few collecting methods, such as sifting and pitfall traps, can be used to sample these overwintering arthropods. The sifting method has a great advantage in collecting higher species richness and individuals of insects than any other trapping method in winter because it enables collection of sedentary and overwintering invertebrates in diapause. In contrast, pitfall traps collect only arthropods that are winter active (Hamilton et al. 2018). As shown by our results, a sifting method combined with a Berlese funnel extraction can be used to effectively collect diverse arthropod taxa in winter, during which the biodiversity of beetles in leaf litter is surprisingly high. Furthermore, a sifting-Berlese funnel method allows sampling of very small invertebrates (less than $1 \mathrm{~mm}$ ) and yields relatively undamaged and rare specimens, thereby providing better opportunities to find new species in this less-explored environment.

Acknowledgements. The authors thank Yeon-Jae Choi, Min-Sang Jang, Jun-Young Kang, Ji-Won Kang, Su-Ho Choi, Tae-Young Jang, and Ji-Wook Kim, who helped collect arthropods in the field, and Dr. Boo-Hee Jung, who helped provide ecological information of Tenebrionidae. They also thank Jeffrey Battigelli and three anonymous reviewers who provided valuable suggestions to improve the manuscript. This study was supported by two grants from the National Institute of Biological Resources (NIBR), funded by the Ministry of Environment (MOE) of the Republic of Korea (NIBR202002112 and NIBR202130202).

Author contributions. U.-J.B. and S.-I.L. contributed equally to this work.

Conflicts of interest. The authors declare that they have no known competing financial interests or personal relationships that could have appeared to influence the work reported in this paper.

Supplementary material. To view supplementary material for this article, please visit https://doi.org/10.4039/tce.2021.54.

\section{References}

Agosti, D., Majer, J.D., Alonso, L.E., and Schultz, T. 2000. Standard methods for measuring and monitoring biodiversity. Smithsonian Institution, Washington, D.C., United States of America. Ahn, K.-J., Cho, Y.-B., Kim, Y.-H., Yoo, I.-S., and Newton, A.F. 2017. Checklist of the Staphylinidae (Coleoptera) in Korea. Journal of Asia-Pacific Biodiversity, 10: 279-336.

Anderson, M., Gorley, R., and Clarke, K.P. 2008. PERMANOVA+ for PRIMER: guide to software and statistical methods. PRIMER-E, Plymouth, United Kingdom.

Andow, D.A. 1991. Vegetational diversity and arthropod population response. Annual Review of Entomology, 36: 561-586.

Anu, A., Sabu, T.K., and Vineesh, P.J. 2009. Seasonality of litter insects and relationship with rainfall in a wet evergreen forest in south western Ghats. Journal of Insect Science, 9: 1-10.

Arnett, R.H., Jr., and Thomas, M.C. 2000. American beetles: Archostemata, Myxophaga, Adephaga, Polyphaga: Staphyliniformia. Volume 1. CRC Press, Boca Raton, Florida, United States of America.

Artz, R., Anastasiou, D., Arrouays, D., Bastos, A.C., Bendetti, A., Bispo, A., et al. 2010. European atlas of soil biodiversity. Publications Office of the European Union, Luxemburg.

Ashford, O.S., Foster, W.A., Turner, B.L., Sayer, E.J., Sutcliffe, L., and Tanner, E.V. 2013. Litter manipulation and the soil arthropod community in a lowland tropical rainforest. Soil Biology and Biochemistry, 62: 5-12. 
Bale, J. and Hayward, S. 2010. Insect overwintering in a changing climate. Journal of Experimental Biology, 213: 980-994.

Bertram, G. 1935. The low temperature limit of activity of Arctic insects. Journal of Animal Ecology, 4: 35-42.

Bouyer, J., Sana, Y., Samandoulgou, Y., Cesar, J., Guerrini, L., Kabore-Zoungrana, C., and Dulieu, D. 2007. Identification of ecological indicators for monitoring ecosystem health in the trans-boundary W Regional park: a pilot study. Biological Conservation, 138: 73-88.

Budka, A., Łacka, A., and Szoszkiewicz, K. 2019. The use of rarefaction and extrapolation as methods of estimating the effects of river eutrophication on macrophyte diversity. Biodiversity and Conservation, 28: 385-400.

Burgess, L. 1981. Winter sampling to determine overwintering sites and estimate density of adult flea beetle pests of rape (Coleoptera: Chrysomelidae). The Canadian Entomologist, 113: 441-447.

Byun, B.-K., Lee, B.-W., Park, S.-Y., Kim, K.-M., and Jo, D.-G. 2009a. Insect fauna of Mt. Sudeogsan in Gyeonggi-do, Korea. Journal of Korean Nature, 2: 155-161.

Byun, B.-K., Lee, B.-W., Park, S.-Y., Lee, Y.-M., and Jo, D.-G. 2009b. Insect fauna of Mt. Nam-san in Seoul, Korea. Journal of Korean Nature, 2: 137-153.

Byun, B.-K., Lim, J.-S., Park, S.-Y., Kim, K.-M., Lee, B.-W., and Jo, D.-G. 2010. Insect fauna of Mt. Gittae-Bong, Samcheok, Gangwon-do. Journal of Korean Nature, 3: 139-149.

Cameron, K.H. and Leather, S.R. 2012. How good are carabid beetles (Coleoptera, Carabidae) as indicators of invertebrate abundance and order richness? Biodiversity and Conservation, 21: 763-779.

Carlton, C. and Robison, H. 1998. Diversity of litter-dwelling beetles in the Ouachita Highlands of Arkansas, USA (Insecta: Coleoptera). Biodiversity and Conservation, 7: 1589-1605.

Chao, A., Chiu, C.H., and Jost, L. 2014a. Unifying species diversity, phylogenetic diversity, functional diversity, and related similarity and differentiation measures through Hill numbers. Annual Review of Ecology, Evolution, and Systematics, 45: 297-324.

Chao, A., Gotelli, N.J., Hsieh, T.C., Sander, E.L., Ma, K.H., Colwell, R.K., and Ellison, A.M. 2014 b. Rarefaction and extrapolation with Hill numbers: a framework for sampling and estimation in species diversity studies. Ecological Monographs, 84: 45-67.

Cho, Y.-B. and Ahn, K.-J. 1999. A review of the subfamily Osoriinae (Coleoptera, Staphylinidae) in Korea. The Korean Journal of Systematic Zoology, 15: 175-181.

Cho, Y.-B. and Ahn, K.-J. 2001. Coleoptera (Silphidae, Staphylinidae). National Institute of Agricultural Science and Technology, Suwon, Korea. [In Korean].

Choi, Y.-J., Park, S.-J., Kim, A-Y., and Park, J.-S. 2020. A new species of the genus Lederina Nikitsky and Belov (Coleoptera: Melandryidae) in Korea. Journal of Asia-Pacific Biodiversity, 13: 720-723.

Clarke, K. and Gorley, R. 2015. Getting started with PRIMER v7. PRIMER-E, Plymouth, United Kingdom.

Cott, H.B. 1940. Adaptive coloration in animals. Oxford University Press, Methuen, United Kingdom.

Dadmal, S. and Khadakkar, S. 2014. Insect faunal diversity collected through light trap at Akola vicinity of Maharashtra with reference to Scarabaeidae of Coleoptera. Journal of Entomology and Zoology Studies, 2: 44-48.

Danks, H.V. 1987. Insect dormancy: an ecological perspective. Biological Survey of Canada (Terrestrial Arthropods), Ottawa, Canada.

De Cáceres, M. and Legendre, P. 2009. Associations between species and groups of sites: indices and statistical inference. Ecology, 90: 3566-3574.

De Cáceres, M., Legendre, P., and Moretti, M. 2010. Improving indicator species analysis by combining groups of sites. Oikos, 119: 1674-1684. 
Deutsch, C.A., Tewksbury, J.J., Huey, R.B., Sheldon, K.S., Ghalambor, C.K., Haak, D.C., and Martin, P.R. 2008. Impacts of climate warming on terrestrial ectotherms across latitude. Proceedings of the National Academy of Sciences, 105: 6668-6672.

Dufrêne, M. and Legendre, P. 1997. Species assemblages and indicator species: the need for a flexible asymmetrical approach. Ecological Monographs, 67: 345-366.

Ferro, M.L., Gimmel, M.L., Harms, K.E., and Carlton, C.E. 2012a. Comparison of the Coleoptera communities in leaf litter and rotten wood in Great Smoky Mountains National Park, USA. Insecta Mundi, 0259: 1-58.

Ferro, M.L., Gimmel, M.L., Harms, K.E., and Carlton, C.E. 2012b. Comparison of three collection techniques for capture of Coleoptera, with an emphasis on saproxylic species, in Great Smoky Mountains National Park, USA. Insecta Mundi, 0261: 1-31.

Fittkau, E.J. and Klinge, H. 1973. On biomass and trophic structure of the central Amazonian rain forest ecosystem. Biotropica, 5: 2-14.

Frazier, M.R., Huey, R.B., and Berrigan, D. 2006. Thermodynamics constrains the evolution of insect population growth rates: "warmer is better". The American Naturalist, 168: 512-520.

Frith, D. and Frith, C. 1990. Seasonality of litter invertebrate populations in an Australian upland tropical rain forest. Biotropica, 22: 181-190.

Gadagkar, R., Chandrashekara, K., and Nair, P. 1990. Insect species diversity in tropics: sampling methods and a case study. Journal of the Bombay Natural History Society, 87: 337-353.

Gilbert, N. and Raworth, D. 1996. Insects and temperature: a general theory. The Canadian Entomologist, 128: 1-13.

Giller, P.S. 1996. The diversity of soil communities, the 'poor man's tropical rainforest'. Biodiversity and Conservation, 5: 135-168.

Hamilton, F. 2015. Phenology and diversity of arthropod communities in leaf litter. University of Arkansas, Fayetteville, North Caroline, United States of America.

Hamilton, F.B., Wiedenmann, R.N., Skvarla, M.J., Sathyamurthy, R., Fisher, D.M., Fisher, J.R., and Dowling, A.P. 2018. Litter-dwelling ground beetles (Coleoptera: Carabidae) and ground spiders (Araneae: Gnaphosidae) of the Ozark Highlands, USA. Southeastern Naturalist, 17: 55-73.

Hammond, P.M. 1992. Global biodiversity, status of the Earth's living resources: a report. Chapman \& Hall, London, United Kingdom.

Hättenschwiler, S., Tiunov, A.V., and Scheu, S. 2005. Biodiversity and litter decomposition in terrestrial ecosystems. Annual Review of Ecology, Evolution, and Systematics, 36: 191-218.

Hayashi, M., Morimoto, K., and Kimoto, S. 1984. The Coleoptera of Japan in color. Volume IV. Hoikusha, Higashiosaka, Japan.

Hébert, C., Jobin, L., Fréchette, M., Pelletier, G., Coulombe, C., Germain, C., and Auger, M. 2000. An efficient pit-light trap to study beetle diversity. Journal of Insect Conservation, 4: 189-200.

Heo, J.-S. and Lee, S.-M. 2015. Vegetation in the area of Pyeongchang and Jeongseon. National Institute of Ecology, Ministry of Environment, Republic of Korea. [In Korean].

Hilty, J. and Merenlender, A. 2000. Faunal indicator taxa selection for monitoring ecosystem health. Biological Conservation, 92: 185-197.

Hoshina, H. and Park, S.-J. 2020. New record of the genus Typhlocolenis Hoshina, 2008 (Coleoptera, Leiodidae) from South Korea with a key to the species. ZooKeys, 991: 129-136.

Hothorn, T., Bretz, F., and Westfall, P. 2008. Simultaneous inference in general parametric models. Biometrical Journal: Journal of Mathematical Methods in Biosciences, 50: 346-363.

Hsieh, T.C., Ma, K.H., and Chao, A. 2016. iNEXT: an R package for rarefaction and extrapolation of species diversity (Hill numbers). Methods in Ecology and Evolution, 7: 1451-1456.

Hughes, J.B., Daily, G.D., and Ehrlich, P.R. 2000. Conservation of insect diversity: a habitat approach. Conservation Biology, 14: 1788-1797.

Janzen, D.H. and Schoener, T.W. 1968. Differences in insect abundance and diversity between wetter and drier sites during a tropical dry season. Ecology, 49: 96-110. 
Jeffery, S. and Gardi, C. 2010. Soil biodiversity under threat: a review. Acta Societatis Zoologicae Bohemicae, 74: 7-12.

Jeong, W.-J. and Ahn, K.-J. 2018. Tachinus (Tachinus) javanus Cameron (Coleoptera: Staphylinidae: Tachyporinae) new to Korea with a key to the known species of the genus Tachinus Gravenhorst. Korean Journal of Applied Entomology, 57: 1-5.

Kai, K.H. and Corlett, R.T. 2002. Seasonality of forest invertebrates in Hong Kong, South China. Journal of Tropical Ecology, 18: 637-644.

Kerekes, J., Kaspari, M., Stevenson, B., Nilsson, R.H., Hartmann, M., Amend, A., and Bruns, T.D. 2013. Nutrient enrichment increased species richness of leaf litter fungal assemblages in a tropical forest. Molecular Ecology, 22: 2827-2838.

Kim, H.-J. and Ahn, K.-J. 2000a. Korean species of the genus Sepeophilus Gistel (Coleoptera, Staphylinidae, Tachyporinae). Insecta Koreana, 17: 251-264.

Kim, H.-J. and Ahn, K.-J. 2000b. A taxonomic review of the genus Tachinus Gravenhorst in Korea (Insecta: Coleoptera: Staphylinidae). Korean Journal of Environmental Biology, 18: 125-131.

Kim, J.-K. and Kwon, O.-C. 2016. Insects fauna of Mt. Yeonin of Gapyeong-gun in Gyeonggi-do. Korean Journal of Nature Conservation, 15: 95-122.

Kim, Y.-J., Min, H.-G., Jeong, S.-W., Ahn, J.-W., and Baek, U.-G. 2017. A guide book of beetles. Aleumwon, Republic of Korea. [In Korean].

Kime, R.D. and Golovatch, S.I. 2000. Trends in the ecological strategies and evolution of millipedes (Diplopoda). Biological Journal of the Linnean Society, 69: 333-349.

Korea Meteorological Administration. 2020. Jeongseon-gun climate record 2011-2019 [online]. Available from https://www.weather.go.kr/w/index.do [accessed 22 April 2020].

Koricheva, J., Mulder, C.P., Schmid, B., Joshi, J., and Huss-Danell, K. 2000. Numerical responses of different trophic groups of invertebrates to manipulations of plant diversity in grasslands. Oecologia, 125: 271-282.

Krishna, M. and Mohan, M. 2017. Litter decomposition in forest ecosystems: a review. Energy, Ecology and Environment, 2: 236-249.

Kurbatov, S.A. 1991. Beetles of the tribe Euplectini (Coleoptera, Pselaphinae) from the Soviet Far East. Communication 2. Zoologicheskiy Zhurnal, 70: 55-62.

Kurosawa, Y., Hisamatsu, S., and Sasaji, H. 1985. The Coleoptera of Japan in color. Volume III. Hoikusha, Higashiosaka, Japan.

Lawton, J., MacGarvin, M., and Heads, P. 1987. Effects of altitude on the abundance and species richness of insect herbivores on bracken. Journal of Animal Ecology, 56: 147-160.

Leather, S.R., Walters, K.F.A., and Bale J.S. 1995. The ecology of insect overwintering. Cambridge University Press, Cambridge, United Kingdom.

Lee, B.-W., Byun, B.-K., Park, S.-Y., Lim, J.-S., and Jo, D.-G. 2009. Insect fauna of Mt. Bongmi-san in Gyeonggi Province, Korea. Journal of Korean Nature, 2: 167-174.

Lee, S.-G. and Ahn, K.-J. 2015. A taxonomic review of Korean species of the Atheta Thomson subgenus Microdota Mulsant \& Rey, with descriptions of two new species (Coleoptera, Staphylinidae, Aleocharinae). ZooKeys, 502: 61-97.

Lee, S.-G. and Ahn, K.-J. 2019. A taxonomic review of Korean Atheta Thomson subgenus Atheta Thomson (Coleoptera, Staphylinidae, Aleocharinae), with descriptions of two new species. Zootaxa, 4613: 305-326.

Lee, S.-I., Jeong, J.-K., Choi, J.-S., and Kwon, O.-K. 2005. Study on community structure and seasonal variations of Coleoptera in Mt. Yeonyeop area, Korea. Korean Journal of Environmental Biology, 23: 71-88.

Legendre, P. and Gallagher, E.D. 2001. Ecologically meaningful transformations for ordination of species data. Oecologia, 129: 271-280.

Legendre, P. and Legendre, L. 2012. Numerical ecology. Elsevier, Amsterdam, The Netherlands. Lim, J.-S., Lee, B.-W., Park, S.-Y., and Jo, D.-G. 2011. Insect fauna of Maebongsan Mountain, Hongcheon-gun, Gangwon-do. Journal of Korean Nature, 4: 293-307. 
Lim, J.-S., Park, S.-Y., Lee, B.-W., and Jo, D.-G. 2012. The insect fauna of Bannonsan Mountain, Jeongseon-gun, Gangwon-do. Journal of Korean Nature, 5: 155-168.

Longcore, T. 2003. Terrestrial arthropods as indicators of ecological restoration success in coastal sage scrub (California, USA). Restoration Ecology, 11: 397-409.

McElravy, E. and Resh, V. 1987. Diversity, seasonality, and annual variability of caddisfly (Trichoptera) adults from two streams in the California coast range. Pascal and Francis Bibliographic, 63: 75-91.

McNabb, D.M., Halaj, J., and Wise, D.H. 2001. Inferring trophic positions of generalist predators and their linkage to the detrital food web in agroecosystems: a stable isotope analysis. Pedobiologia, 45: 289-297.

Mellanby, K. 1939. Low temperature and insect activity. Proceedings of the Royal Society of London. Series B: Biological Sciences, 127: 473-487.

Moore, J.C., Walter, D.E., and Hunt, H.W. 1988. Arthropod regulation of micro- and mesobiota in below-ground detrital food webs. Annual Review of Entomology, 33: 419-439.

Ødegaard, F. 2000. How many species of arthropods? Erwin's estimate revised. Biological Journal of the Linnean Society, 71: 583-597.

Oksanen, J., Blanchet, F.G., Friendly, M., Kindt, R., Legendre, P., McGlinn, D., et al. 2019. Vegan: community ecology package. R package version 2.5-6. Available from cran.r-project.org/ package $=$ vegan [accessed on 26 May 2020]

Olson, D.M. 1994. The distribution of leaf litter invertebrates along a Neotropical altitudinal gradient. Journal of Tropical Ecology, 10: 129-150.

Park, G.-H. and Cho, S.-W. 2007. Comparison of insect diversity in relation to the sampling method, time and window. Korean Journal of Applied Entomology, 46: 375-383.

Park, K.-T., Kwon, Y.-J., Park, J.-K., Bae, Y.-S., Bae, Y.-J., Byun, B.-K., et al. 2012. Insect of Korea. GeoBook, Seoul, Republic of Korea. [In Korean].

Park, S.-J., Cho, Y.-H., Kim, Y.-J., Han, Y.-G., Lim, H.-M., Park, S.-K., and Hong, E.-J. 2010. Insect Fauna of Is. Boleum-do (Prov. Gyeonggi-do), Korea. Journal of Korean Nature, 3: 15-24.

Park, S.-Y., Lim, J.-S., Jo, D.-G., and Lee, B.-W. 2011. Insect fauna of (Mt.) Daemisan, Gangwon Province, Korea. Journal of Korean Nature, 4: 87-97.

Pianka, E.R. and Parker, W.S. 1975. Ecology of horned lizards: a review with special reference to Phrynosoma platyrhinos. Copeia, 1975: 141-162.

Pinheiro, F., Diniz, I., Coelho, D., and Bandeira, M. 2002. Seasonal pattern of insect abundance in the Brazilian cerrado. Austral Ecology, 27: 132-136.

R Core Team. 2020. R: a language and environment for statistical computing. R Foundation for Statistical Computing, Vienna, Austria. Available from http://www.R-project.org/ [accessed 11 November 2020].

Recher, H., Majer, J., and Ganesh, S. 1996. Seasonality of canopy invertebrate communities in eucalypt forests of eastern and western Australia. Australian Journal of Ecology, 21: 64-80.

Redford, K.H. 1987. Ants and termites as food. In Current mammalogy. Edited by H.H. Genoways. Springer, New York, New York, United States of America. Pp. 349-399.

Rees, C.J.C. 1983. Microclimate and the flying Hemiptera fauna of a primary lowland rain forest in Sulawesi. In Tropical rainforest: ecology and management. Edited by S.L. Sutton, T.C. Whitmore, and A.C. Chadwick. Blackwell Scientific Publications, Oxford, United Kingdom. Pp. 121-136.

Régnière, J., Powell, J., Bentz, B., and Nealis, V. 2012. Effects of temperature on development, survival and reproduction of insects: experimental design, data analysis and modeling. Journal of Insect Physiology, 58: 634-647.

Robinson, M.H. and Robinson, B. 1970. Prey caught by a sample population of the spider Argiope argentata (Araneae: Araneidae) in Panama: a year's census data. Zoological Journal of the Linnean Society, 49: 345-358. 
Robinson, S.I., McLaughlin, Ó.B., Marteinsdóttir, B., and O’ Gorman, E.J. 2018. Soil temperature effects on the structure and diversity of plant and invertebrate communities in a natural warming experiment. Journal of Animal Ecology, 87: 634-646.

Rosenzweig, M.L. 1995. Species diversity in space and time. Cambridge University Press, Cambridge, United Kingdom.

Roy, S., Roy, M., Jaiswal, A., and Baitha, A. 2018. Soil arthropods in maintaining soil health: thrust areas for sugarcane production systems. Sugar Tech, 20: 376-391.

Ruggiero, A., Sackmann, P., Farji-Brener, A.G., and Kun, M. 2009. Beetle abundanceenvironment relationships at the subantarctic-Patagonian transition zone. Insect Conservation and Diversity, 2: 81-92.

Ruppert, E.E., Barnes, R.D., and Fox, R.S. 2004. Introduction to Arthropoda. In Invertebrate zoology: a functional evolutionary approach. Edited by E.E. Ruppert, R.D. Barnes, and R.S. Fox. Thomson-Brooks/Cole, Belmont, California, United States of America. Pp. 523-524.

Saji, A. and Al Dhaheri, S.S. 2014. Diversity and seasonality of some of the ground-dwelling invertebrates in the eastern region of Abu Dhabi, United Arab Emirates. International Journal of Biodiversity and Conservation, 6: 271-279.

Sánchez-Reyes, U.J., Niño-Maldonado, S., and Jones, R.W. 2014. Diversity and altitudinal distribution of Chrysomelidae (Coleoptera) in Peregrina Canyon, Tamaulipas, Mexico. ZooKeys, 417: 103-132.

Santos, S.A., Cabanas, J.E., and Pereira, J.A. 2007. Abundance and diversity of soil arthropods in olive grove ecosystem (Portugal): effect of pitfall trap type. European Journal of Soil Biology, 43: 77-83.

Saunders, D.S. 2002. Insect clocks, 3rd edition. Elsevier, Amsterdam, The Netherlands.

Scherber, C., Mwangi, P.N., Temperton, V.M., Roscher, C., Schumacher, J., Schmid, B., and Weisser, W.W. 2006. Effects of plant diversity on invertebrate herbivory in experimental grassland. Oecologia, 147: 489-500.

Scott, J.A. and Epstein, M.E. 1987. Factors affecting phenology in a temperate insect community. American Midland Naturalist, 117: 103-118.

Seastedt, T.R. and Crossley, D.A., Jr. 1984. The influence of arthropods on ecosystems. BioScience, 34: $157-161$.

Siddig, A.A., Ellison, A.M., Ochs, A., Villar-Leeman, C., and Lau, M.K. 2016. How do ecologists select and use indicator species to monitor ecological change? Insights from 14 years of publication in Ecological Indicators. Ecological Indicators, 60: 223-230.

Smythe, N. 1973. Biological monitoring data-insects. In Environmental monitoring and base-line data. Edited by R.W. Rubinoff. Smithsonian Institution Environmental Science Program, Washington, D.C., United States of America. Pp. 70-115.

Tauber, M.J. and Tauber C.A. 1976. Insect seasonality: diapause maintenance, termination, and postdiapause development. Annual Review of Entomology, 21: 81-107.

Tauber, M.J., Tauber, C.A., and Masaki, S. 1986. Seasonal adaptations of insects. Oxford University Press, New York, United States of America.

Taylor, L. 1963. Analysis of the effect of temperature on insects in flight. Journal of Animal Ecology, 32: 99-117.

Ueno, S.I., Kurosawa, Y., and Sato, M. 1985. The Coleoptera of Japan in color. Volume II. Hoikusha, Higashiosaka, Japan.

Ulyshen, M.D. and Hanula, J.L. 2009. Litter-dwelling arthropod abundance peaks near coarse woody debris in loblolly pine forests of the southeastern United States. Florida Entomologist, 92: 163-164.

Vasconcellos, A., Andreazze, R., Almeida, A.M., Araujo, H.F., Oliveira, E.S., and Oliveira, U. 2010. Seasonality of insects in the semi-arid Caatinga of northeastern Brazil. Revista Brasileira de Entomologia, 54: 471-476. 
Venables, W.N. and Ripley, B.D. 2002. Modern applied statistics with S, 4th edition. Springer, New York, United States of America.

Wagner, T.L., Wu, H.I., Feldman, R.M., Sharpe, P.J., and Coulson, R.N. 1985. Multiple-cohort approach for simulating development of insect populations under variable temperatures. Annals of the Entomological Society of America, 78: 691-704.

Wardhaugh, C., Stone, M., and Stork, N. 2018. Seasonal variation in a diverse beetle assemblage along two elevational gradients in the Australian wet tropics. Scientific Reports, 8: 1-12.

Whittaker, J.B. and Tribe, N.P. 1998. Predicting numbers of an insect (Neophilaenus lineatus: Homoptera) in a changing climate. Journal of Animal Ecology, 67: 987-991.

Wolda, H. 1978a. Fluctuations in abundance of tropical insects. The American Naturalist, 112: $1017-1045$.

Wolda, H. 1978b. Seasonal fluctuations in rainfall, food and abundance of tropical insects. Journal of Animal Ecology, 47: 369-381.

Wolda, H. 1980. Seasonality of tropical insects. Journal of Animal Ecology, 49: 277-290.

Wolda, H. 1987. Altitude, habitat and tropical insect diversity. Biological Journal of the Linnean Society, 30: 313-323.

Wolda, H. 1988. Insect seasonality: why? Annual Review of Ecology and Systematics, 19: 1-18.

Wolda, H. and Broadhead, E. 1985. Seasonality of Psocoptera in two tropical forests in Panama. Journal of Animal Ecology, 54: 519-530.

Work, T.T., Buddle, C.M., Korinus, L.M., and Spence, J.R. 2002. Pitfall trap size and capture of three taxa of litter-dwelling arthropods: implications for biodiversity studies. Environmental Entomology, 31: 438-448.

Cite this article: Byeon, U.-J., Lee, S.-I., Park, S.-J., Kim, A-Y., and Park, J.-S. 2021. Winter sampling and seasonal variation in litter-dwelling beetle assemblages using a sifting method. The Canadian Entomologist. https://doi.org/10.4039/tce.2021.54. 